\title{
Nonlinearity induced destruction of resonant tunneling in the Wannier-Stark problem
}

\author{
S. Wimberger ${ }^{1}$, R. Mannella ${ }^{1}$, O. Morsch ${ }^{1}$, E. Arimondo ${ }^{1}$, A.R. Kolovsky ${ }^{2,3}$, and A. Buchleitner ${ }^{2}$ \\ ${ }^{1}$ Dipartimento di Fisica E. Fermi, CNR-INFM, Unversità di Pisa, Largo Pontecorvo 3, 56127 Pisa, Italy \\ ${ }^{2}$ Max-Planck-Institut für Physik komplexer Systeme, Nöthnitzer Str. 38, 01187 Dresden, Germany \\ ${ }^{3}$ Kirensky Institute of Physics, 660036 Krasnoyarsk, Russia
}

(Dated: November 11, 2018)

\begin{abstract}
We present detailed numerical results on the dynamics of a Bose-Einstein condensate in a tilted periodic optical lattice over many Bloch periods. We show that an increasing atom-atom interaction systematically affects coherent tunneling, and eventually destroys the resonant tunneling peaks.
\end{abstract}

PACS numbers: 03.75.Lm,03.65.Xp,05.60.Gg

Experiments with cold and ultracold atoms made it possible in the last decade to prepare and control the centre-of-mass motion of atoms with unprecedented precision. Many toy models of either many-body solid state physics 1, 2, 3, 4, 5] or of simple Hamiltonian systems, whose complexity arises from an external driving force [6], were realized with the exceptional control offered by static or time-dependent optical potentials.

Particularly Bose-Einstein condensates (BEC) whose initial momentum spread can be adjusted in width and absolute position have proved to be an extremely helpful experimental tool [3, 4, 7, 8, 9]. In addition, a BEC offers interesting new features originating from the intrinsic interactions between the atoms. Examples of such effects are new quantum phases [10], soliton-like motion [11], the occurrence of energetic or dynamical instabilities in condensates [7, 9, 12, 13], or the decay and subsequent revival of Bloch oscillations (BO) [14].

We focus on the evolution of a BEC loaded into a onedimensional lattice and subjected to an additional static force $F$, which is most easily realized and controlled by accelerating the optical lattice [1, 3]. In previous experiments, a BEC was accelerated to allow for a single crossing of the Brillouin zone (BZ), and two effects were observed: for large accelerations, an enhanced tunneling probability from the ground state band to the first excited band due to the atom-atom interaction was measured [3, 8]. Secondly, for smaller accelerations (where tunneling is negligible) signatures of a dynamical instability in the BEC were observed [9, 15]. By contrast, here we investigate the dynamics of a BEC performing many Bloch oscillations (BO), and we ask ourselves how the atom-atom interaction affects tunneling for a sequence of BZ crossings. In particular, we scan $F$ to study the impact of the atom-atom interaction on resonantly enhanced tunneling (RET), for which the standard LandauZener prediction is modified even in the absence of interactions 16]. The RET leads to a faster decay of the Wannier states trapped in the potential wells. With the survival probability and the recurrence probability (see Eqs. (4) and (6) below) we present two consistent measures for the nonlinear RET which define clear experimental signatures of the destruction of the coherent tunneling process inside the periodic potential.

If we neglect interactions for a moment, our system will be described by the Hamiltonian

$$
H=-\frac{\hbar^{2}}{2 M} \frac{d^{2}}{d x^{2}}+V \sin ^{2}\left(\frac{\pi x}{d_{L}}\right)+F x
$$

Here $d_{L}$ is the spatial period of the optical lattice with maximal amplitude $V$, and $M$ the atomic mass. Eq. (1) defines the well-known Wannier-Stark problem, which gives rise to $\mathrm{BO}$ with period $T_{\mathrm{Bloch}}=h / d_{L} F$ ( $h$ is Planck's constant). If tunneling is small, we can view the system as moving at a constant speed in momentum space within the fundamental BZ. At the zone edge, most of the wave packet is reflected (giving rise to $\mathrm{BO}$ ) while a small part can tunnel across the first band gap to the next higher-lying energy band and then escape quickly by successive tunneling events across the smaller (higher) band gaps. Landau-Zener theory predicts a decay rate [16]

$$
\Gamma(F) \propto F e^{-\frac{b}{F}}
$$

where $b$ is proportional to the square of the energy gaps. Eq. (2) is modified by RET which occurs when two Wannier-Stark levels in neighboring potential wells are coupled strongly due to their accidental degeneracy. The RET results in pronounced peaks in the tunneling rates, e.g., as a function of $1 / F$, on top of the global exponential decay described by (2) 16]. In this paper we investigate the impact of the effective shift of the Wannier-Stark levels by a nonlinear interaction term.

For the linear problem (1), the decay rates have been measured previously in the regime of short life-times in the ground state band (of the order of $100 \mu \mathrm{s}$ ), where $\Gamma(F)$ is essentially smooth [17]. Since RET is a coherent quantum effect, the peaks should be sensitively affected by the atom-atom interaction, which can be varied experimentally by changing either the density of the BEC or through the atom-atom scattering potential via a Feshbach resonance [18]. Our results are a consequence of many sequential Landau-Zener events, and they show the destruction of a RET peak with increasing interaction strength, in a regime which is experimentally accessible.

We use a fully 3D Gross-Pitaevskii equation (GPE) [19] to describe the temporal evolution of a BEC which 
is subject to realistic potentials:

$$
\begin{gathered}
\mathrm{i} \hbar \frac{\partial}{\partial t} \psi(\vec{r}, t)=\left[-\frac{\hbar^{2}}{2 M} \nabla^{2}+\frac{1}{2} M\left(\omega_{x}^{2} x^{2}+\omega_{\mathrm{r}}^{2} \rho^{2}\right)+\right. \\
\left.V \sin ^{2}\left(\frac{\pi x}{d_{L}}\right)+F x+g N|\psi(\vec{r}, t)|^{2}\right] \psi(\vec{r}, t) .
\end{gathered}
$$

$\psi(\vec{r}, t)$ represents the condensate wave function, and the frequencies $\omega_{x}$ and $\omega_{\mathrm{r}}$ characterize the longitudinal and transverse harmonic confinement (here with cylindrical symmetry: $\rho=\sqrt{y^{2}+z^{2}}$ ). We fixed $d_{L}=1.56 \mu \mathrm{m}$ and $V / E_{\mathrm{R}}=5$ for our computations, with the recoil energy $E_{\mathrm{R}}=p_{\mathrm{R}}^{2} / 2 M$ for $p_{\mathrm{R}}=\hbar \pi / d_{L}$, and the recoil period $T_{\mathrm{R}}=h / E_{\mathrm{R}}$. The above values for $d_{L}$ and $V$ were realized in the experiments reported in [3, [8, [9] based on two laser beams propagating at an angle different from $\pi$. In Eq. (3), the nonlinear coupling constant is given by $g=4 \pi \hbar^{2} a_{s} / M$, where $a_{s}$ is the $s$-wave scattering length and $N$ the number of atoms in the BEC [19, 20]. The dimensionless nonlinearity $C=g n_{0} /\left(8 E_{\mathrm{R}}\right)$ is computed from the peak density of the initial state of the condensate, with $C=0.027 \ldots 0.31$ for the experimentally investigated range of [3], and with $C=0.5$ reached in [21]. Here we focus on $C>0$, but report briefly also on attractive interactions with $C<0$. The latter case leads to a fundamentally different behavior of the system because the collapse of the condensate introduces an additional time scale, which for experimentally relevant parameters is of the order of $10 \mathrm{msec}$ [18, 22] (slightly longer than $T_{\text {Bloch }}=1.8 \ldots 3.0 \mathrm{msec}$ here).

The GPE (3) is numerically integrated using finite difference propagation, adapted by a predictor-corrector estimate to reliably evaluate the nonlinear interaction [19]. Since our system is essentially the problem of a constantly accelerated particle for the part of the wave function which has tunnelled out of the first BZ already, one must be careful with the application of absorbing boundary conditions or complex coordinate methods 23, 24]. To avoid any spurious effects due to the fast spreading, we use a large numerical basis. In this way, we fully cover the $3 \mathrm{D}$ expansion of the entire wave packet, including its tunnelled tail, without the use of non-Hermitian potentials. The initial state propagated by (3) is the relaxed condensate wave function, adiabatically loaded into the confining potential given by the harmonic trap and the optical lattice (with $F=0$ ). Approximate analytic forms of the relaxed state are found, e.g., in [25], but we used an imaginary time propagation to reliably compute the initial state for $C>0$.

The linear decay rates for non-interacting atoms in the optical lattice are computed from the spectrum of the $1 \mathrm{D}$ Wannier-Stark problem of Eq. (10) using, e.g., the method of [16]. Those linear rates are plotted in Fig. 1 The maxima in the rates occur when $F d_{L} m$ (with $m$ integer) is close to the difference between the first two energy bands (averaged over the BZ) of the $F=0$ problem [16]. The actual peaks are slightly shifted with respect to the above estimate (marked by arrows in the inset of Fig. 1), owing to a field-induced level shift [16].

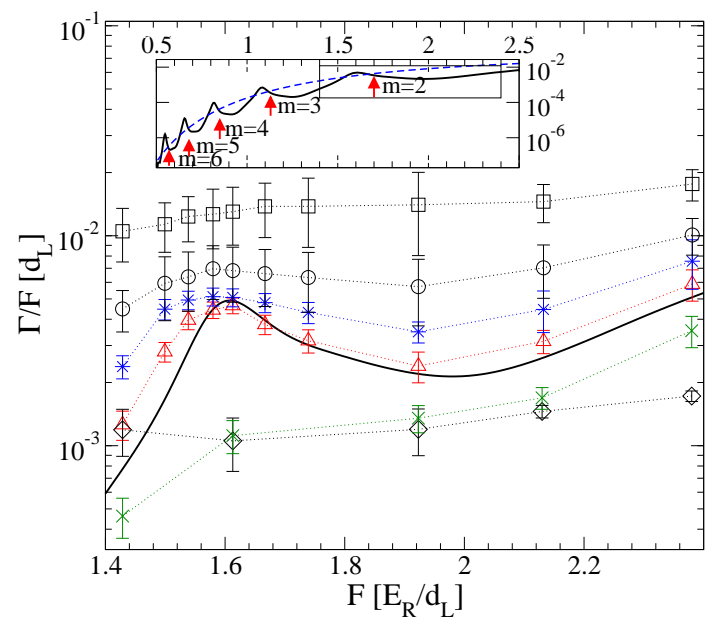

FIG. 1: (color online). Tunneling rates obtained by exponential fits to data of $P_{\text {sur }}(t)$ as the solid-line fits in Fig. 3 Here the peak in the box in the inset is scanned locally, while globally the rates follow an exponential law (dashed line in the inset). $C=-0.31$ (diamonds), $C=-0.065$ (crosses), $C=0$ (solid line), $C=0.027$ (pyramids), $C=0.065$ (stars), $C=0.12$ (circles), $C=0.31$ (squares). The "error" bars interpolate different exponential fits such as the dot-dashed ones in Fig. 3 The arrows in the inset mark the peak positions as predicted by the simple argument stated in the text.

Experimentally, the most easily measurable quantity is the momentum distribution of the BEC obtained from a free expansion after the evolution inside the lattice. From the momentum distribution we determine the survival probability by projection of the evolved state $\psi(\vec{p}, t)$ onto the support of the initial state

$$
P_{\text {sur }}(t) \equiv \int_{-p_{\mathrm{c}}}^{p_{\mathrm{c}}} d p_{x}\left(\int d p_{y} d p_{z}|\psi(\vec{p}, t)|^{2}\right)
$$

where $p_{\mathrm{c}} \geq 3 p_{\mathrm{R}}$ is a good choice since three momentum peaks are initially significantly populated, corresponding to $-2 p_{\mathrm{R}}, 0,2 p_{\mathrm{R}}[3,25]$.

Figure 2 shows the initial population in momentum space (inset in (a)) as compared with the population after 10 BO periods, for both the linear and the nonlinear case. The increase of $C>0$ has two effects: firstly, it enhances the tunneling for the first few crossings of the BZ. Secondly, it scrambles the out-coupled part of the wave function (see Fig. 2 and its complement in Fig. 4 below), as previously observed in [2, 3, 5]. The change in the momentum distributions after various Landau-Zener events is a manifestation of the intrinsic instability of the nonlinear GPE dynamics [9, 12].

Instead of studying the details of the distributions shown in Fig. 2] we will focus on the temporal decay of the survival probability in the following. Figure 3 presents $P_{\text {sur }}(t)$, which for the linear case has an exponential form (apart from the $t \rightarrow 0$ limit [26])

$$
P_{\text {sur }}(t) \sim e^{-\frac{t \Gamma}{\hbar}},
$$




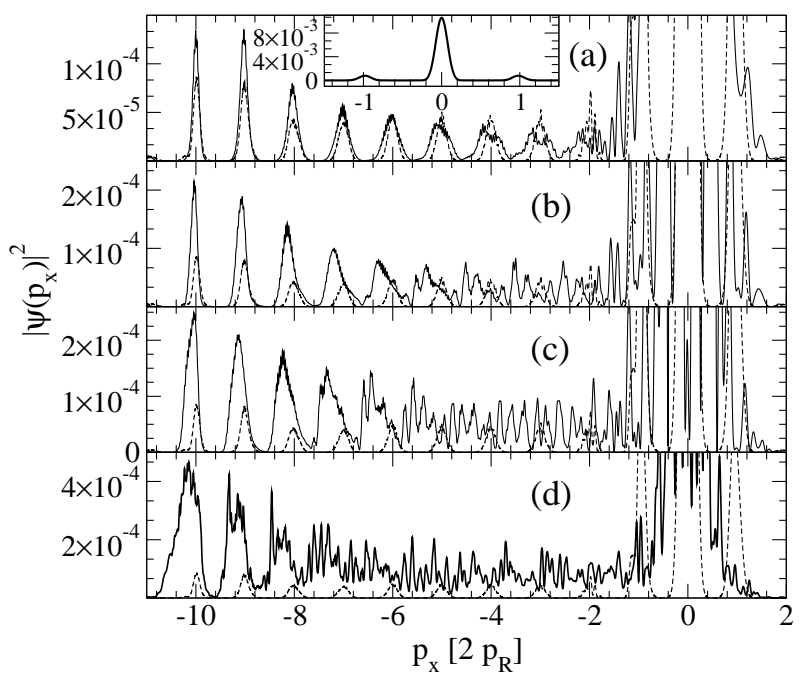

FIG. 2: Momentum distributions after 10 BOs, for $T_{\mathrm{R}} / T_{\text {Bloch }}=1.428 . \quad C=0$ (dotted) compared with (a) $C=0.027$ (full line; the inset shows the corresponding $t=0$ distribution), (b) $C=0.065$, (c) $C=0.12$, and (d) $C=0.31$.

with the characteristic exponent $\Gamma$. The temporal behavior of $P_{\text {sur }}$ depends significantly on $C$. For $C= \pm 0.31$, we observe clear deviations from a purely exponential decay, as present for small $C$. A repulsive nonlinearity initially enhances the tunneling more than after about five crossings of the BZ (see fits to data in Fig. 3). This deviation from the mono-exponential behavior means that the tunneling events occurring at different integer multiples of the Bloch period are correlated by the presence of the nonlinearity. Since the remaining density becomes smaller, the impact of the nonlinearity becomes less. The result is that the rate $\Gamma$ is defined only locally in time, and its value systematically decreases as time increases.

An attractive interaction can stabilize the system at the RET peak, which is shown for $C=-0.31$ in Fig. 3(b). For optimal comparison, we chose the same initial state (for $C=+0.31$ ) which then was evolved for $F \neq 0$ with $C=-0.31$. Such a scenario could be realized by a sudden change of the sign of the scattering length through a Feshbach resonance [18]. This result is consistent with studies of simpler models, where a resonance state can be stabilized at system-specific strengths of the nonlinearity [24, 27].

The impact of the nonlinearity on the dynamical evolution of the "closed" system confined to the fundamental BZ can be studied with the help of the recurrence probability [17], defined by the autocorrelation

$$
P_{\text {rec }}(t) \equiv|\langle\psi(t) \mid \psi(t=0)\rangle|^{2} .
$$

The BO manifest themselves as the periodic oscillations

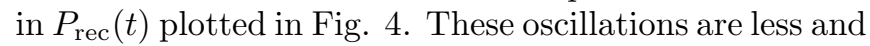
less pronounced with increasing $C$, in much the same way as the momentum peaks are washed out when the band edge is crossed in the regime of instability [9]. In contrast

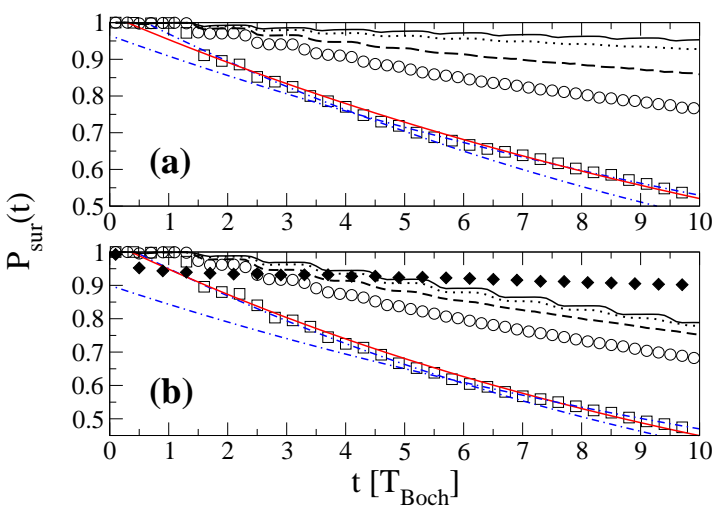

FIG. 3: (color online). $\quad P_{\text {sur }}(t)$ for (a) $T_{\mathrm{R}} / T_{\text {Bloch }}=1.428$ and (b) 1.613 (the peak maximum in Fig. 1). $C$ is scanned from -0.31 (diamonds in (b) only), 0 (solid line), 0.027 (dotted line), 0.065 (dashed line), 0.12 (circles) to 0.31 (squares). The gray/red solid lines show global exponential fits to the $C=0.31$ data, while the dot-dashed lines show exponential fits for small and large $t$ respectively. From those fits, the rates in Fig. 1 and their systematical variation in time are obtained. The step-like structures reflect the periodic BO and are correlated with the dephased oscillations in Fig. 4

to the survival probability, $P_{\text {rec }}$ is a phase sensitive measure, and therefore it shows - in addition to the temporal decay - the dephasing of the BO due to the nonlinearity. For $C=0$, the recurrence maxima decay in time with the same rate as $P_{\text {sur }}(t)$, which offers an alternative method for extracting $\Gamma$. For $C \neq 0, P_{\text {rec }}$ can be integrated over time, and the rates are extractable by the approximate proportionality between the integrated area and the inverse decay rate (recalling that $\int d t f(t) \exp (-t \Gamma) \sim 1 / \Gamma$ to leading order, for a periodic function $f(t)$ ). The latter approach works because we can determine the linear rate from a direct fit to $P_{\text {rec }}$ and then compare the ratio of the linear and the nonlinear area (denoted by $A_{0}$ and $A_{C}$ ). This rough estimate $\Gamma_{C} \sim \Gamma_{0} A_{0} / A_{C}$ agrees within $25 \%$ with the rate extracted from the fits to the data of Fig. 3 The estimate could be improved if we knew the analytic form of the function $f(t)$, and it breaks down for large $C$, when the periodic oscillations in $P_{\text {rec }}$ are destroyed.

Having introduced two methods to extract the tunneling rates, we scan the parameter $F$ across a RET peak of the globally exponential curve $\Gamma(1 / F)$ (see Fig. 1). The scanned range in $F$ corresponds to values of lattice accelerations between $0.99 \mathrm{~ms}^{-2}$ and $1.65 \mathrm{~ms}^{-2}$, which are standard in experiments [3, 9].

A repulsive nonlinearity particularly affects the wings of the peak and, for small $C$, much less the peak maximum. The global increase of $\Gamma$ with increasing $C$ is qualitatively predicted in [28], with enhanced single LandauZener crossing probabilities induced by the effective reduction of the energy gap due to the nonlinearity. The left and right-most points in Fig. 10 are in the regime where an amended version of (2) indeed applies [28], and here $\Gamma / F$ is approximately proportional to $C$. However, near the peak, the rates do not follow a simple scaling 


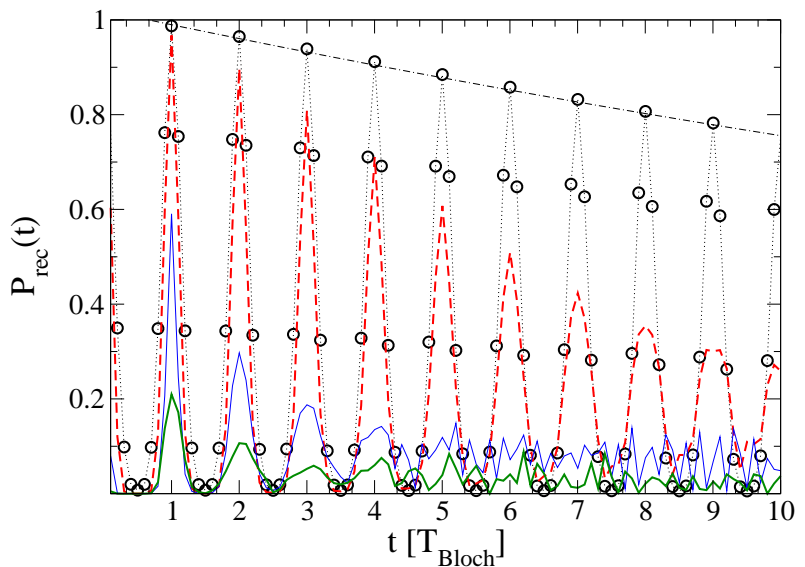

FIG. 4: (color online). $P_{\text {rec }}(t)$ for the data shown in Fig. 31 b) with $C=0$ (circles), 0.027 (dashed), 0.12 (thin line), and 0.31 (thick line). The dot-dashed line presents an exponential fit to the maxima of the $C=0$ data.

law as a function of $C$, and the argumentation of 28] does not apply.

For $C<0$ we also observe the destruction of the RET peak. For $C=-0.065$, the BEC clearly stabilizes in the potential wells, whilst for $C=-0.31$ the situation is more complicated (see Fig. (1). The precise dynamics of the system is governed by the two separate time scales for tunneling and collapse, which strongly depend on parameters in the sensitive RET regime.

In an experiment, $\omega_{x}$ can either be set to zero or decreased to $\omega_{x} / 2 \pi \lesssim 1 \mathrm{~Hz}$ to realize a quasi $1 \mathrm{D}$ nonlinear
Wannier-Stark problem. We verified that letting $\omega_{x}$ tend to zero for the evolution with $F \neq 0$, or applying a small finite $\omega_{x}$ gives the same results for the BO cycles studied here. Furthermore, for $0<C \lesssim 0.05$, using the renormalized nonlinearity of [20] we observed that a $1 \mathrm{D}$ version of Eq. (3) reproduces well the 3D data. If $|C|$ is larger, the nonlinearity couples the longitudinal and transverse degrees of freedom, which affects the dynamics of a real $\mathrm{BEC}$ in a non-trivial way [19]. The $1 \mathrm{D}$ computations are feasible up to 100 Bloch periods, and this would allow one to extract the tunneling rates more reliably. The effect of the nonlinearity is, however, hardly visible for $0<C<0.05$, and quantitative predictions for a broad range of $C$ relied on 3D computations.

To summarize, we observed and quantified the deformation and destruction of the RET peaks due to interactions in a BEC in an accelerated optical lattice. Our results complement ongoing studies of interaction-induced processes such as dynamical instabilities or the decay and subsequent revival of $\mathrm{BO}$. In the regime of small nonlinearity, where dynamical instabilities are not fully developed, the survival and recurrence probabilities experience an exponential decay modified by the condensate nonlinearity. The temporal decay of these observables remains a useful indicator also for large nonlinearity, even if the resonant structure in the tunneling rate is washed out.

We thank M. Cristiani and D. Ciampini for helpful discussions and the Humboldt Foundation (Feodor-Lynen Program), MIUR COFIN-2004, and ESF (QUDEDIS) for support.
[1] M. BenDahan et al., Phys. Rev. Lett. 76, 4508 (1996); S.R. Wilkinson et al., ibid. 76, 4512 (1996).

[2] B.P. Anderson and M.A. Kasevich, Science 282, 1686 (1998).

[3] O. Morsch et al., Phys. Rev. Lett. 87, 140402 (2001); M. Cristiani et al., Phys. Rev. A 65, 063612 (2002).

[4] J. Hecker-Denschlag et al., J. Phys. B 35, 3095 (2002).

[5] G. Roati et al., Phys. Rev. Lett. 92, 230402 (2004).

[6] M.G. Raizen, Adv. At. Mol. Opt. Phys. 41, 43 (1999); D.A. Steck, W.H. Oskay, and M.G. Raizen, Science 293, 274 (2001); W.K. Hensinger et al., Nature (London) 412, $52(2001)$.

[7] L. Fallani et al., Phys. Rev. Lett. 93, 140406 (2004).

[8] M. Jona-Lasinio et al., Phys. Rev. Lett. 91, 230406 (2003).

[9] M. Cristiani et al., Opt. Express 12, 4 (2004).

[10] M. Greiner et al., Nature (London) 415, 39 (2002).

[11] B. Eiermann et al., Phys. Rev. Lett. 92, 230401 (2004).

[12] B. Wu and Q. Niu, New J. Phys. 5, 104 (2003).

[13] Y. Zheng, M. Kostrun, and J. Javanainen, Phys. Rev. Lett. 93, 230401 (2004).

[14] A.R. Kolovsky, Phys. Rev. Lett. 90, 213002 (2003); A. Buchleitner and A.R. Kolovsky, ibid., 91, 253002 (2003); Q. Thommen, J.C. Garreau, and V. Zehnle, ibid., 91 210405 (2003).
[15] Instability at the band edge has also been studied using a different experimental protocol in 7].

[16] M. Glück, A.R. Kolovsky, and H.J. Korsch, Phys. Rev. Lett. 83, 891 (1999); Phys. Rep. 366, 103 (2002).

[17] C.F. Bharucha et al., Phys. Rev. A 55, R857 (1997).

[18] J.L. Roberts et al., Phys. Rev. Lett. 86, 4211 (2001).

[19] E. Cerboneschi et al., Phys. Lett. A 249, 495 (1998); S. Wimberger et al., Phys. Rev. Lett. 94, 130404 (2005).

[20] M. Olshanii, Phys. Rev. Lett. 81, 938 (1998).

[21] N. Gemelke et al., cond-mat/0504311

[22] S. Wüster, I.J. Hope, and C.M. Savage, Phys. Rev. A 71, 033604 (2005).

[23] T. Paul, K. Richter, and P. Schlagheck, Phys. Rev. Lett. 94, 020404 (2005).

[24] P. Schlagheck and T. Paul, cond-mat/0402089 N. Moiseyev and L.S. Cederbaum, cond-mat/0406189

[25] P. Pedri et al., Phys. Rev. Lett. 87, 220401 (2001).

[26] S.R. Wilkinson et al., Nature (London) 387, 575 (1997).

[27] D. Witthaut, S. Mossmann, and H.J. Korsch, J. Phys. A 38, 1777 (2005).

[28] D.I. Choi and Q. Niu, Phys. Rev. Lett. 82, 2022 (1999); O. Zobay, B.M. Garraway, Phys. Rev. A 61, 033603 (2000). 